\title{
Retos del periodismo frente a las redes sociales, las fake news y la desconfianza de la generación $\mathbf{Z}$
}

\section{Challenges for journalism facing social networks, fake news and the distrust of $\mathrm{Z}$ Generation}

Ana Pérez Escoda. Universidad Antonio de Nebrija. España. aperezes@nebrija.es

$[\underline{\mathrm{CV}}] \odot \mathrm{G}$ ( $)$

Luis Miguel Pedrero Esteban. Universidad Antonio de Nebrija. España.

lpedrero@nebrija.es

$[\mathrm{CV}]$ G $\mathrm{G}$

Este trabajo forma parte del Proyecto Horizon 2020 "Mediatized Discourses on Europeanization and their Representations in Public Perceptions" MEDIATIZAED.EU [N $\left.{ }^{\circ} 101004534\right]$ (H2020-SC6-Transformations2020), financiado por la Comisión Europea (Inicio:01/01/2021 - Finalización: 01/01/2023).

Cómo citar este artículo / Referencia normalizada

Pérez Escoda, A. y Pedrero Esteban, L.M. (2021). Retos del periodismo frente a las redes sociales, las fake news y la desconfianza de la generación Z. Revista Latina de Comunicación Social, 79, 67-85. https://www.doi.org/10.4185/RLCS-2021-1519

\begin{abstract}
RESUMEN
Introducción: Las redes sociales se han erigido en el canal preferente de los jóvenes para conocer la actualidad pese a haberse convertido en un flujo incontrolado de informaciones que tienden a polarizar las opiniones, a alimentar las controversias y a sembrar la desconfianza. Medios y periodistas tratan de definir nuevos modelos comunicativos adaptados a estos espacios virtuales donde puedan defender su legitimidad y contrarrestar el impacto de una desinformación cada vez más extendida. Este trabajo se centra en el estudio de la confianza, el consumo y la percepción de los jóvenes españoles hacia los medios y las fake news sobre la premisa de que el conocimiento de tales indicadores contribuirá a recuperar la esencia del periodismo. Metodología: A partir de una metodología cuantitativa de carácter descriptivo y exploratorio, se aplica un cuestionario a una población de 465 jóvenes entre 18 y 24 años. El análisis se centra en dos constructos de estudio: 1) uso y consumo de medios y redes sociales; y 2) recepción, distinción y percepción de las fake news. Resultados: Se evidencia una desconfianza abierta de la generación $\mathrm{Z}$ hacia a los medios, las redes, los políticos y los periodistas. La gran paradoja es que, aun admitiendo su recelo hacia las redes sociales, los jóvenes no renuncian a su consumo masivo. Discusión y conclusiones: La adecuación entre la información que se comunica y la realidad, así como la conexión con las expectativas de quienes la reciben, se impone como una necesidad sustancial para el periodismo en el reto de legitimar su discurso en las redes sociales.
\end{abstract}


PALABRAS CLAVE: redes sociales; periodismo; fake news; jóvenes; generación Z; desconfianza; comunicación.

\begin{abstract}
Introduction: Social networks have become in the current crisis scenario not only the channel most consumed by young people, but also an uncontrolled flow of information that tends to polarize opinions, fuel controversies and sow distrust. The media -and with them journalists- must define new communicative models adapted to these virtual spaces where they can recover their legitimacy and counteract the problematic expansion of disinformation. This paper focuses on the study of trust, consumption and perception of young Spaniards towards the media and fake news, on the premise that knowledge of audiences will help professionals to recover the essence of journalism. Methodology: Based on a descriptive and exploratory quantitative methodology, a questionnaire was applied to a population of 465 young people between 18 and 24 years old. The analysis focuses on two study constructs: 1) use and consumption of media and social networks; and 2) reception, distinction and perception of fake news. Results: The results show an open distrust of Generation $\mathrm{Z}$ towards media, networks, politicians and journalists. The great paradox is that, admitting their distrust towards networks, young people declare their massive consumption. Discussion and conclusions: The adequacy between the information that is communicated and reality, as well as the connection with the expectations of those who receive it, stands as a substantial and unpostponable challenge for journalism in the challenge of legitimizing its discourse in social networks.
\end{abstract}

KEYWORDS: social media; journalism; fake news; youth; Z generation; distrust; communication.

\title{
CONTENIDO
}

1. Introducción. 1.1. Redes sociales y desinformación: dos caras de la misma moneda. 1.2. Medios, redes y jóvenes. 2. Objetivos. 3. Metodología. 3.1. Muestra de estudio. 4. Resultados. 4.1. Consumo de medios y redes sociales entre los jóvenes. 4.2. Consumo, recepción y percepción de fakenews. 5. Conclusiones y Discusión. 6. Bibliografía 7. Currículum Vitae

\section{Introducción}

Los medios de comunicación, concebidos como actores sociales legitimados para contar y trasladar al gran público el relato de la actualidad, se han visto destronados en las últimas décadas por la irrupción de dos factores determinantes: por un lado, la eclosión de las redes sociales como sustitutos digitales que han sabido ganar terreno en rapidez, cantidad, frecuencia y presencia (Dessart, 2017; Masip et al., 2017; Pérez et al., 2020), además de introducir a los usuarios como creadores activos de contenido (Larrondo et al., 2020; Castillo-Abdul et al., 2020); y, por otro, el desarrollo devastador de una "era de la posverdad" (Keyes, 2004) en la que el binomio inseparable de políticos y medios ha propiciado discursos basados en la apelación emocional para influir en la opinión, utilizando como recurso las herramientas que brindan las redes (Viner, 2016): clickbaiting, instantaneidad, algoritmos, filtros burbuja, personalización de la información, etc. Las posibilidades infinitas de impacto y alcance que mostraban las redes parecían una solución ante la pérdida paulatina de las audiencias más jóvenes, que han naturalizado estas ventanas como medios nativos de interacción y socialización (Sádaba y Pérez-Escoda, 2020). Durante años, numerosos medios han invertido en estrategias multicanal, presencia en redes, contenidos específicos, ediciones web con nuevas usabilidades, etc.; sin embargo, esa apuesta no ha logrado frenar la pérdida progresiva de audiencias jóvenes (Larrondo et al, 2020). El panorama actual plantea serias exigencias para un periodismo desgastado, desacreditado y poco consumido por aquellos que, navegando por las redes, ya no aceptan las estrategias de un discurso mediático lastrado por sus propias debilidades: la posverdad 
RLCS, Revista Latina de Comunicación Social, 79, 67-85

[Investigación] DOI: 10.4185/RLCS-2021-1519| ISSN 1138-5820| Año 2021

engañó durante un tiempo, pero ¿está funcionando como se esperaba en las generaciones más jóvenes?

\subsection{Redes sociales y desinformación: dos caras de la misma moneda}

Ambos fenómenos han evolucionado de manera paralela en las dos primeras décadas del siglo XXI. Las redes han impuesto la plataformización de la información y la comunicación debido a su imparable penetración en la población: ocho de cada diez españoles entre 16 y 65 años ya disponen de, al menos, un perfil en alguno de estos espacios virtuales, que durante 2020 -el año del confinamiento a causa del coronavirus- sumaron ocho nuevos millones de usuarios hasta alcanzar la cifra de 37 millones (We Are Social, 2021). La llegada de las redes sociales en la primera década del nuevo milenio supuso el comienzo de una confluencia continua que autores como Jenkins denominaron convergencia mediática (Jenkins, 2008). La irrupción y progresiva normalización de las redes sociales dio lugar a una cierta controversia ante su rol híbrido como vehículos de participación e interacción de la audiencia, pero también canales de distribución de contenidos de los medios tradicionales. Ante el cambio en las pautas de consumo mediático progresivamente mediatizadas por el smartphone, el streaming y las propias interacciones en los escenarios virtuales de participación e interacción (Gutiérrez García y Barrios Rubio, 2021), la prensa, la radio y la televisión no sólo dejaron de ser fuente preferente para informarse, sino también para dar forma a la opinión pública de la ciudadanía. Como apuntan Casero-Ripollés y García-Gordillo, "su autoridad se deteriora y su influencia social se diluye; además, están perdiendo la centralidad en la conversación política en las redes sociales, con la consiguiente reducción de su capacidad de influencia en ese escenario" (2020: 157).

Por su lado, la llamada 'era de la posverdad' ha ido calando en la sociedad y transformando "de forma invisible el mundo que experimentamos mediante el control de lo que vemos y lo que no vemos" (Pariser, 2011: 88). La desinformación como técnica de persuasión ha ido extendiéndose en un entorno digital donde resulta más fácil que nunca difundir informaciones o desinformaciones de modo masivo y con efecto inmediato. El enorme potencial de los efectos sobre las redes de las noticias falsas quedó demostrado en la campaña presidencial de EE UU en 2016, que con el empleo de los algoritmos marcó un antes y un después para los medios y los políticos. El resultado es un complejo desorden de la información en el que las noticias creíbles y confiables compiten con diferentes tipos de material falso y engañoso; la desinformación se ha convertido en una de las amenazas más peligrosas durante la crisis por la pandemia de la COVID-19, especialmente por su facilidad y rapidez para extenderse a través de las redes sociales y por el alcance que allí encuentran en el público que las utiliza con mayor frecuencia: los jóvenes (Masip et al., 2020).

La desinformación, descrita por la Comisión Europea (2018) como la "información falsa, imprecisa o engañosa, presentada y promovida para obtener ingresos o causar daño público intencionadamente", resulta una elocuente amenaza al protagonismo de medios y periodistas. La Organización Mundial de la Salud, por su parte, ya había alertado antes de la pandemia de los peligros de la infodemia, cuyo significado se equipara a la "sobreabundancia de información sobre un tema, ya sea rigurosa y veraz o falsa y confusa, que dificulta a la gente encontrar fuentes de confianza" (WHO, 2020). En cuanto al término fake news, elegido en 2017 como palabra del año en los diccionarios británicos Collins y Oxford, se describe como la información tergiversada, a menudo sensacionalista y difundida bajo la apariencia de noticia, y -en combinación con las redes sociales- ha resultado ser verdadera cicuta para el ejercicio y los fines del Periodismo (Farkas y Schou, 2019; Elías, 2018).

En Europa se trabaja desde 2015 para luchar contra la desinformación ante la preocupación que supone la proliferación de fake news y la amenaza a las sociedades democráticas. El Eurobarómetro 
de otoño de 2019 (European Commission, 2020) ya reflejaba una mayor preocupación de los españoles $(86 \%)$ frente a los europeos $(74 \%)$; ese mismo informe señala que la desconfianza hacia las redes sociales es mayor en España (73\%) que en el resto el continente (65\%). En este contexto de sobreabundancia informativa y de descentralización de la información es donde con mayor facilidad se distribuyen las denominadas fake news, informaciones falsas que, con apariencia de noticias periodísticas, se difunden deliberadamente para promover intereses particulares y que inducen a error a los lectores (Aruguete et al., 2020).

Los principales focos de producción de noticias falsas son las redes sociales y las páginas webs. Según Banks (2020), por estos canales su difusión es más extensa, efectiva y profunda que las informaciones veraces debido a las reacciones que suscitan y a la rapidez de alcance con el público. Si antes de la crisis mundial por la COVID-19 la desinformación suponía un obstáculo importante para la libertad de información de la ciudadanía, la pandemia aceleró este problema por la ansiedad que se generó a causa del encierro. No obstante, el efecto de la desinformación difiere en cada sociedad y territorio dependiendo del nivel de educación, la cultura democrática, la confianza en las instituciones, la inclusividad de los sistemas electorales, la importancia del dinero en los procesos políticos y las desigualdades sociales y económicas (Pérez-Curiel y Velasco Molpeceres, 2020).

\subsection{Medios, redes y jóvenes: donde tres son multitud}

La proliferación de estas noticias constituye un problema para toda la ciudadanía, pero afecta de modo particular al público más joven, el que más emplea las redes sociales como vías de conexión con la actualidad (Mendiguren, Pérez-Dasilva y Meso-Ayerdi, 2020). Este nicho poblacional absorbe de forma especialmente intensa el determinismo tecnológico y la conectividad propias del ecosistema digital contemporáneo: las ventajas de la usabilidad, la accesibilidad, la multimedialidad y el lenguaje hipertextual alcanzan su máxima plenitud en las redes sociales (Sádaba y Pérez-Escoda, 2020), donde al hecho de que las noticias estén continuamente disponibles se une a la posibilidad de que las puedan compartir de forma inmediata, aportando incluso sus propias valoraciones.

Analizando el panorama informativo se aprecia un cambio de tendencia: las generaciones más jóvenes no solo han naturalizado el consumo de información en redes ignorando a los medios tradicionales, sino también su participación en ellas para dar lugar a una transformación directa en el propio proceso: el "periodismo ciudadano" (Espiritusanto y Gonzalo Rodríguez, 2011; Paulussen et al., 2008). Los medios comienzan así a perder su capacidad de informar, pues la instantaneidad de las redes se impone con carácter global y las mejoras tecnológicas hacen que las noticias alcancen al usuario casi en tiempo real. La información está en otros lugares y se produce por otros canales y en formatos y lenguajes distintos. Los jóvenes de la generación $\mathrm{Z}$ escriben los borradores de la historia, la eligen y transforman; el storytelling ya no es potestativo de medios o periodistas: ahora cada usuario tiene la palabra.

En este contexto, los medios sociales parecen sustituir a los medios de comunicación tradicionales, cada vez presentes en más segmentos poblacionales (Gómez Calderón et al., 2020; Pérez-Maíllo et al; Pérez-Escoda et al., 2020). Si bien en el informe Digital News Report 2019 ya se descubría que hasta un 38\% de los españoles de entre 18 y 24 años preferían las redes sociales y los blogs para obtener información (40\% en el estudio de 2020), con la llegada de la crisis del coronavirus y el aumento de los bulos asociados al estado de crispación, la edición de 2020 constata un descenso de la confianza de la ciudadanía en los medios de comunicación y apenas llega al 36\%, la cifra más baja en cinco años. 
Resulta evidente que la interacción entre comunicación, tecnología y sociedad está condicionando la construcción y distribución de los discursos en el nuevo milenio (Álvarez-Ramos, Heredia-Ponce y Romero, 2018); ha llegado a su fin el concepto de masa que sustentaba a los medios clásicos (Jarvis, 2015), y han emergido nuevos hábitos de consumo ciudadano basados en la inmediatez, la reducción en la longitud y profundidad de los mensajes, la interacción y la autoafirmación de creencias previas. Todo ello ha derivado en una pérdida de confianza de las audiencias más jóvenes hacia los periodistas y los medios tradicionales, y sin esa legitimidad pierde relevancia la función social del Periodismo: cualquier actor social puede ahora acceder a las plataformas digitales para hacer circular contenidos y convertirse así en fuente informativa para otros (Paulussen et al., 2008). Se ha pasado de un modelo vertical, con una fuerte jerarquía de los medios como gestores y gatekeapers, a otro horizontal cada vez más descentralizado, abierto e híbrido (Loosen et al., 2020), donde conviven -a veces de forma armónica y otras conflictiva- los viejos y nuevos medios profesionales, por un lado, y los amateurs, por otro. Estos últimos alcanzan eco principalmente a través de las redes sociales, espacios donde también se ejercen la atención y la influencia, pero donde el control de la información veraz ya no es responsabilidad de nadie.

La globalidad y el alcance de las redes sociales supone de un tiempo a esta parte la oportunidad de los medios para alcanzar a unas audiencias que migran masivamente de consumos tradicionales a entornos virtuales, planteando retos y oportunidades (Romero-Rodríguez et al., 2021), pero también riesgos (Larrondo et al., 2020;). El fenómeno de las redes ha alterado la naturaleza de la comunicación pública y privada (Van Dijck, 2016), alcanzando su pináculo de éxito cuando la marca se convierte en verbo (googlear, tuitear, etc.). Este fenómeno rompe el tradicional estudio de la comunicación, añadiendo al escenario comunicativo nuevos agentes que, legitimados por el público en general y por el contexto digital en particular, se alzan con contundencia por la confianza que generan y el impacto que logran transformando el entorno comunicativo (Barrios y Pedrero-Esteban, 2021; Pérez et al., 2020).

Y así, pese a la cuestionable y contraproducente capacidad de los medios digitales para generar bulos y fakes news -más aún en tiempos de crisis (Boulianne y Theocharis, 2020)-, se impone la reacción aplastante de seguidores en redes que potencian un caudal informativo-comunicativo sin precedentes. La participación de la ciudadanía en los medios digitales como iguales -mediada por la intervención de las compañías tecnológicas (Galloway, 2018)- y la generación de un diálogo global interconectado, otorgan un valor incuestionable a estas ágoras digitales, que desplazan el discurso comunicativo y consiguen -como en el ámbito político- que la participación de los jóvenes se vera fortalecida gracias a su participación en redes sociales (Castillo-Abdul et al., 2020). De ahí la pertinencia del estudio aquí planteado, que trata de identificar la dimensión y los efectos de este nuevo "modus operandi" de los jóvenes al aproximarse a la actualidad informativa: a la fuentes, al tratamiento, a su credibilidad y a su criterio crítico al valorar los riesgos de la exposición a las redes sociales como referencias sobre las que conocer lo que sucede en su entorno de proximidad -en sentido físico pero, sobre todo, emocional- y, a partir de ese conocimiento, tomar conciencia y postura propia con relación a las personas, hechos e instituciones que les rodean.

\section{Objetivos}

Como ya se ha descrito, la problemática que supone el fenómeno de las fake news va asociada a un cambio en el acceso a la información por parte los jóvenes, que están sustituyendo los medios por las redes sociales, ámbitos donde aquellos todavía no han asentado un discurso legitimador. Así, resulta fundamental ahondar en un aspecto sociológico clave en esta generación cuyo desarrollo cognitivo va indisociablemente unido a las dinámicas hiperconectadas del ecosistema online: el conocimiento de sus sentimientos y percepciones, las motivaciones de sus usos y consumos en el controvertido 
entorno de la desinformación. Por ello se justifica la investigación desarrollada en este artículo, cuyo objetivo principal es realizar un acercamiento exploratorio y descriptivo sobre el consumo de redes sociales y medios, así como de las percepciones que sobre las fake news albergan los jóvenes españoles desde el comienzo de la crisis por la COVID-19. Con el fin de profundizar en este objetivo se han establecido las siguientes preguntas de investigación:

P.I.1. ¿El consumo de medios por parte de los jóvenes está relacionado con el grado de fiabilidad que otorgan a los medios?

P.I.2. ¿Se sienten informados con el consumo de medios que hacen? ¿Tiene relación esta percepción con el tiempo que le dedican?

P.I.3. ¿Son capaces de distinguir las fake news? Si es así, ¿son conscientes de recibirlas?

P.I.4. ¿Es el fact cheking una opción contra las fake news para esta generación?

P.I.5. ¿Qué medios asocian más con la distribución de las noticias falsas?

P.I.6. ¿Qué contenidos se asocian más a las noticias falsas?

P.I.7. ¿Los medios y los periodistas generan confianza entre esta generación?

\section{Metodología}

El enfoque metodológico del estudio es de corte cuantitativo y específicamente exploratorio; se utiliza para ello una estadística descriptiva que no busca hacer inferencias, sino relación entre variables. Como técnica de recolección de datos se empleó el cuestionario, por ser la más idónea en este tipo de estudios (Vilches, 2012). Para su diseño se tomaron como modelo dos ya existentes: Media Use in the European Union (European Commission, 2019) y Digital News Report.es 2020 (Negredo et al., 2020), que sirvieron para adaptar el instrumento final de la investigación. Su elaboración siguió dos etapas: 1) los investigadores articularon los ítems en tres bloques atendiendo a la tipología de variables de estudio: a) sociodemográficas, b) relacionadas con el uso de medios y redes sociales, y c) consumo, recepción y percepción de fake news. Los dos últimos bloques se corresponden con los constructos de estudio (Tabla 1) definidos para identificar una serie de comportamientos intangibles de la muestra, tomando como referencia los trabajos previos de Couldry et al. (2007) y Pérez-Tornero (2020).

Tabla 1. Definición de constructos de estudio, variables y número de ítems

\begin{tabular}{|c|c|c|c|}
\hline Definición teórica de constructos & Constructos & Variables de estudio & Ítems \\
\hline \multirow{13}{*}{$\begin{array}{l}\text { Aceptación de un modelo que afecta la } \\
\text { construcción de la propia identidad a } \\
\text { partir de la exposición a informaciones } \\
\text { y estímulos audiovisuales en un } \\
\text { tiempo, un lugar y a través de unos } \\
\text { dispositivos o medios físicos. Esto } \\
\text { implica asumir puntos de vista, } \\
\text { razonamientos, estéticas y valores para } \\
\text { una construcción cultural (Couldry, } \\
\text { Livingstone, and Markha, 2007; Pérez- } \\
\text { Tornero, 2020). }\end{array}$} & \multirow{6}{*}{$\begin{array}{l}\text { C1. Consumo de } \\
\text { medios y redes } \\
\text { sociales }\end{array}$} & $\mathrm{V}_{1}:$ Medios más consumidos & 8 \\
\hline & & $\mathrm{V}_{2}:$ Percepción fiabilidad & 8 \\
\hline & & $\mathrm{V}_{3}$ : Redes sociales más usadas & 8 \\
\hline & & $\mathrm{V}_{4}:$ Tiempo de consumo & 1 \\
\hline & & $\mathrm{V}_{5}$ : Percepción de estar informado & 1 \\
\hline & & $\mathrm{V}_{6}:$ Sentimiento-redes-Covid-19 & 1 \\
\hline & \multirow{7}{*}{$\begin{array}{l}\text { C2. Consumo, } \\
\text { recepción y } \\
\text { percepción de } \\
\text { fake news }\end{array}$} & $\mathrm{V}_{1}$ : Info más consumida desde Covid-19 & 8 \\
\hline & & $\mathrm{V}_{2}$ : Capacidad de distinguir fake news & 8 \\
\hline & & $\mathrm{V}_{3}:$ Recepción fake & 1 \\
\hline & & $\mathrm{V}_{4}:$ Conocimiento y uso de fact checkers & 1 \\
\hline & & $\mathrm{V}_{5}$ : Medios asociados a la distribución de fake & 9 \\
\hline & & $\mathrm{V}_{6}:$ Contenidos con más fake news & 3 \\
\hline & & $\mathrm{V}_{7}:$ Sentimiento de confianza con fuentes & 8 \\
\hline
\end{tabular}

Fuente: Elaboración propia

En un segundo proceso el cuestionario fue enviado a un panel de expertos $(\mathrm{N}=5)$ que valoraron la pertinencia y claridad del instrumento a partir de los constructos de estudio y las variables 
establecidas. El resultado de esta evaluación supuso reducir el número inicial de ítems del cuestionario (de 120 ítems a 105) por redundancia o falta de idoneidad, así como la reformulación de ocho ítems que se percibían confusos. Terminado el proceso de diseño del instrumento, se procedió al estudio de fiabilidad y consistencia a través del alfa de Cronbach con unos resultados de 0.79 y 0.82 , respectivamente, en la media de las variables de cada constructo, lo cual implica valores aceptables ( $\geqq 0.7)$ en su consistencia (Tavakol y Dennick, 2011). La caracterización de las variables de estudio se centró en las categorías cualitativas de carácter ordinal, para las que se utilizó una escala tipo Likert con cinco valores en las que 1 equivalía a "nada o nunca" y 5 a "mucho o todo", y variables cualitativas de tipo nominal donde los valores numéricos asignados no presentan escala de valor, sino codificación numérica. Por razones de espacio, en este estudio se presentan resultados parciales de la investigación.

\subsection{Muestra de estudio}

La técnica de muestreo empleada en este trabajo fue no probabilística y no aleatoria, también conocida como muestreo de conveniencia, combinada con la técnica "bola de nieve" para facilitar la recolección de datos en un momento en el que las distancias sociales y la falta de alumnado presencial en las aulas universitarias dificulta la labor de los investigadores en la recolección de datos. Así pues, en un primer estadio se compartió el cuestionario vía online, utilizando la herramienta Google Forms; aprovechando la proximidad de los investigadores con la muestra objeto de estudio, se pidió a los sujetos la máxima difusión entre sus círculos.

Las características de la investigación, que buscaba exclusivamente sujetos en una cohorte de edad determinada (entre 18 y 24 años), hizo que estas técnicas resultaran idóneas, pues se trataba de un muestreo selectivo. La muestra la forman 465 estudiantes en grados universitarios de Comunicación y Educación, todos comprendidos entre 18 y 24 años, es decir, pertenecientes a la llamada Generación $\mathrm{Z}(\mathrm{M}=21,5 \mathrm{DT}=0.907$; Min.=18; Máx.=25). La distribución por género estuvo determinada como sigue: género femenino $\mathrm{N}=244(52,47 \%)$ y género masculino $\mathrm{n}=221(47,52 \%)$. A su vez, y debido al tipo de muestreo, se recogieron datos de diferentes Comunidades Autónomas, como se muestra en el Gráfico 1.

Antes de rellenar la encuesta, cada sujeto fue informado y dio su consentimiento para la utilización de los datos con fines exclusivamente académicos y anónimos. Tras la recolección en el programa Excel, se realizó la codificación y la creación de la base de datos para el análisis de los resultados en el paquete estadístico SPSS. Para la aproximación descriptiva y exploratoria se han utilizado frecuencias, medias, desviaciones típicas y correlación entre variables según las características de dichas variables. 


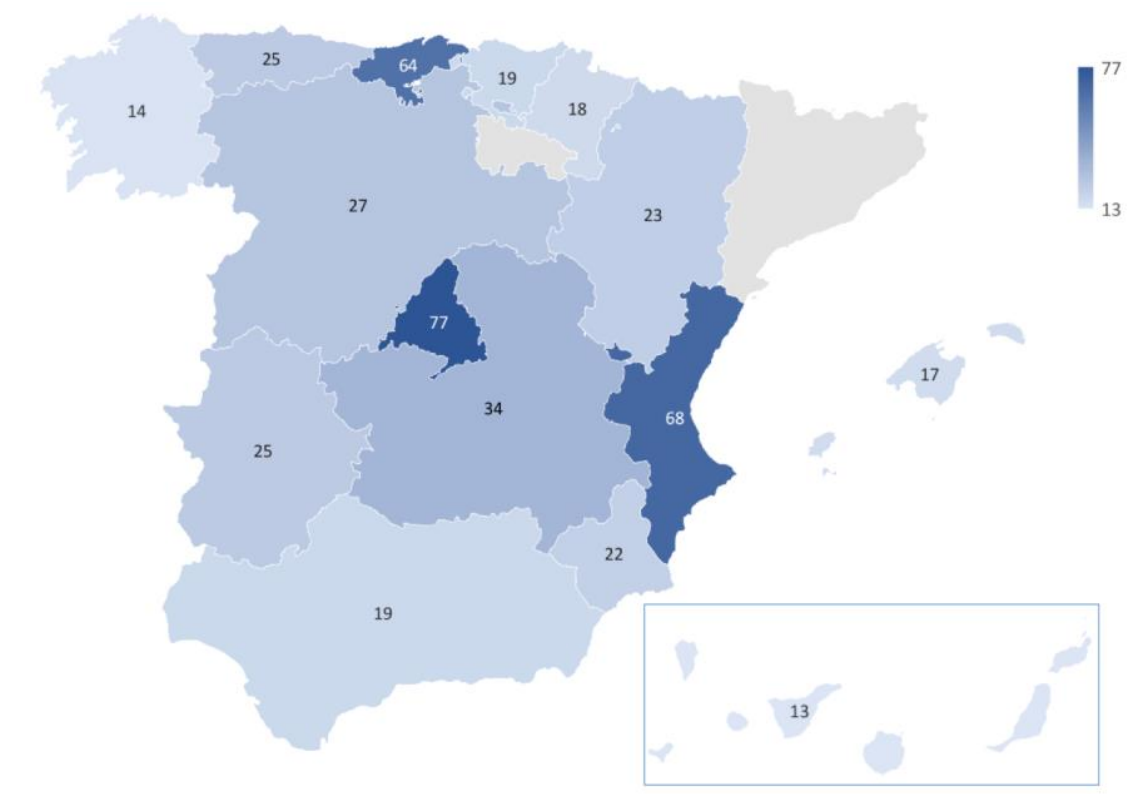

\section{Gráfico 1. Distribución de la muestra por Comunidades Autónomas}

Fuente: Elaboración propia a partir de la muestra

\section{Resultados}

La presentación de los resultados se realiza en dos fases correspondientes a los dos constructos de estudio: C1. Consumo de medios y redes sociales (con un total de seis variables y 27 ítems de estudio) y C2. Consumo, recepción y percepción de fake news (con un total de siete variables y 38 ítems).

\subsection{Consumo de medios y redes sociales entre los jóvenes}

En el estudio de este constructo subyace un modelo complejo y estructurado en el que el acto de consumir medios conduce a intangibles -especificados en las variables de estudio- y determina una radiografía de los hábitos de consumo en una población que, como se ha definido previamente, tiene características determinadas. En este sentido, los primeros datos que se presentan hacen referencia a la primera variable de consumo de medios.

Tabla 2. Estadísticos descriptivos para el análisis de las variables $V_{l}$

\begin{tabular}{|c|c|c|c|c|c|c|c|c|}
\hline & Media & DT & $\begin{array}{c}\text { Nunca } \\
(\%)\end{array}$ & $\begin{array}{c}\text { Poco } \\
(\%)\end{array}$ & $\begin{array}{c}\text { Ocasion } \\
\text { al }(\%)\end{array}$ & $\begin{array}{c}\text { Con } \\
\text { frecuencia } \\
(\%)\end{array}$ & $\begin{array}{c}\text { Siempre } \\
(\%)\end{array}$ & $\mathbf{N}$ \\
\hline $\mathrm{V}_{1}$ : Radio (analógica) & 1,75 & 0,907 & 49 & 32,9 & 12,5 & 4,7 & 0,9 & 465 \\
\hline $\mathrm{V}_{1}:$ Radio online & 1,6 & 0,848 & 59,6 & 25,4 & 11 & 3,9 & 0,2 & 465 \\
\hline $\mathrm{V}_{1}:$ Prensa & 1,83 & 0,926 & 44,9 & 34,4 & 14,4 & 5,4 & 0,9 & 465 \\
\hline $\mathrm{V}_{1}$ : Televisión online & 2,19 & 1,215 & 38,3 & 25,4 & 21,3 & 8,8 & 6,2 & 465 \\
\hline $\mathrm{V}_{1}:$ Prensa digital & 3,44 & 1,082 & 4,5 & 14,2 & 32 & 31 & 18,3 & 465 \\
\hline $\mathrm{V}_{1}:$ Televisión & 3,54 & 1,125 & 5,2 & 13,5 & 25,4 & 34,2 & 21,7 & 465 \\
\hline $\mathrm{V}_{1}$ : Páginas web & 3,67 & 1,018 & 3,4 & 9 & 26 & 40,2 & 21,3 & 465 \\
\hline $\mathrm{V}_{1}$ : Redes sociales & 4,25 & 0,976 & 1,9 & 4,3 & 13,8 & 27,3 & 52,7 & 465 \\
\hline
\end{tabular}

* $V_{l}$ : Habitualmente te informas a través de...

Fuente: Elaboración propia 
Los resultados de la variable $\left(\mathrm{V}_{1}\right)$ "Habitualmente te informas a través de..." ofrecen una primera impresión sobre el consumo que los sujetos de estudio hacen de los medios para informarse. Se muestra una tendencia central muy marcada en los que han contestado que "Nunca" se informan a través de la radio, con un porcentaje del $49 \%(\mathrm{M}=1,75$; $\mathrm{DT}=0,907)$; la radio online, con un $59,6 \%$ $(\mathrm{M}=1,6 ; \mathrm{DT}=0,848)$; la prensa, con un 44,9\% $(\mathrm{M}=1,83$; $\mathrm{DT}=0,926)$; y la televisión online, con un 38,3\% ( $M=2,19 ; \mathrm{DT}=1,215)$. La tendencia se invierte en los otros cuatro medios analizados: si sumamos los resultados obtenidos para los valores "Con frecuencia" y "Siempre", los resultados son altos para la prensa online, con un porcentaje de 48,3\% (M=3,44; $\mathrm{DT}=1,082)$; para la televisión, con un 55,9\% (M=3,54; DT=1,125); y para las páginas web, con un 61,5\% (M=3,67; $\mathrm{DT}=1,018)$. En el caso de las redes sociales, los datos son muy altos, con un $79 \%$ de los encuestados $(\mathrm{M}=4,25$; DT= $0,976)$.

Profundizando en los resultados, en el análisis de la segunda variable $\left(\mathrm{V}_{2}\right)$ "Grado de fiabilidad que te merecen los medios" encontramos que la inercia de las respuestas tiene una tendencia opuesta, como se observa en el Gráfico 2 , donde se correlacionan ambas variables $\left(\mathrm{V}_{1}-\mathrm{V}_{2}\right)$. Si sumamos los resultados de los valores más bajos ("Ninguna o poca credibilidad"), observamos que los medios menos fiables para la muestra de estudio son, por orden de mayor a menor credibilidad: la televisión online, con un 30,3\% (M=3,01; DT=0,934); las páginas web, con un 45,1\% (M=2,69; DT=0,845); y el medio que menos credibilidad obtiene son las redes sociales con un $58,7 \%(\mathrm{M}=2,42$; DT=0,86). Por el contrario, si sumamos los valores más altos de la codificación "Credibilidad alta y muy alta", los resultados más altos son, por orden creciente: radio online con un 41,1\% (M=3,25; $\mathrm{DT}=0,795)$; la TV, con un 43,8\% (M=3,01; DT=0,934); la prensa digital, con un 44,3\% (M=3,34; $\mathrm{DT}=0,868)$; la radio analógica, con un 51,4\% $(\mathrm{M}=3,43$; $\mathrm{DT}=0,815)$; y la prensa, con un $58,5 \%(\mathrm{M}=3,57$; $\mathrm{DT}=$ $0,874)$.

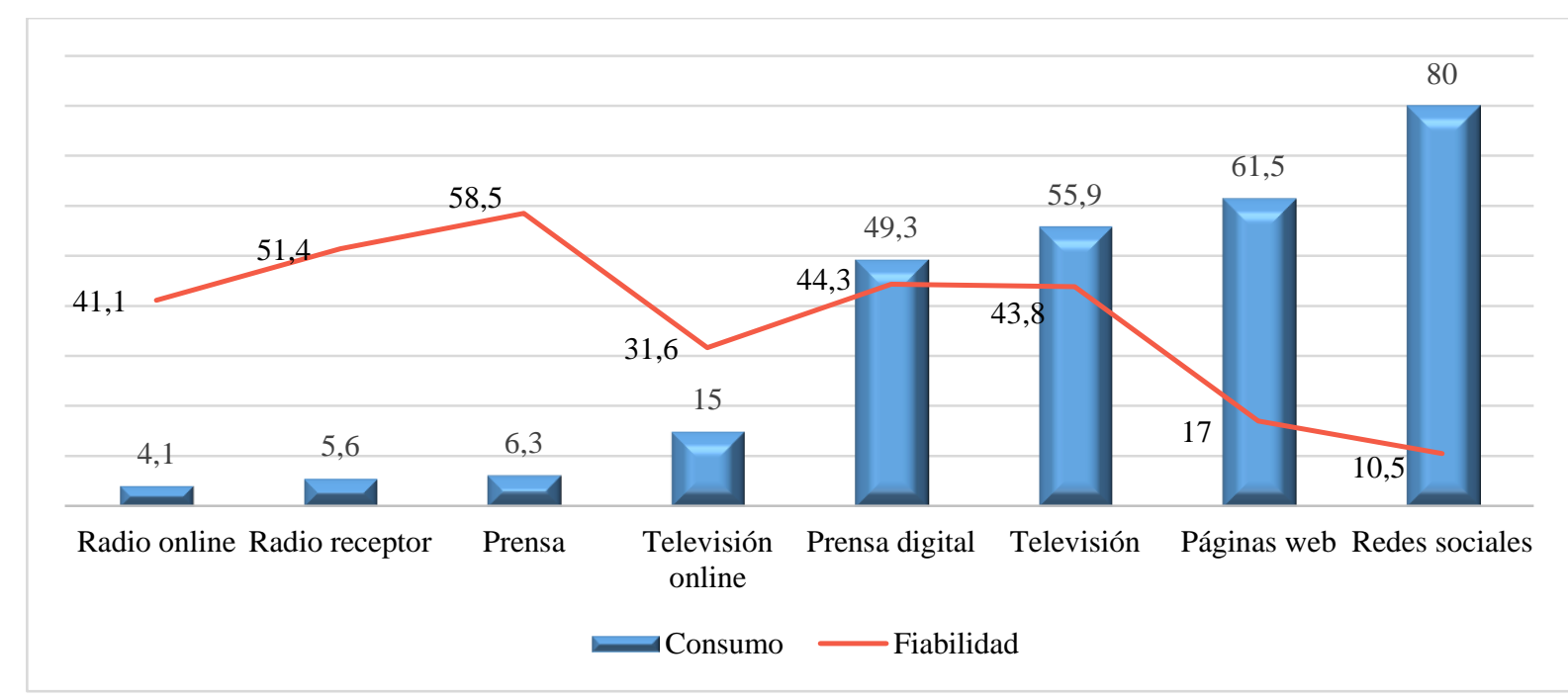

Gráfico 2. Correlación entre medios más consumidos y grado de fiabilidad otorgado a los medios Fuente: Elaboración propia a partir de la muestra

Estos datos suponen una evolución en las percepciones sociales: si hace unos años se demostraba que la falta de confianza en los medios se había trasladado hacia las redes sociales, consumidas de manera masiva -así lo indicaban varios estudios en países europeos como los de Newman y Fletcher (2017), Nielsen y Graves (2017) o Webster (2014)-, la falta de confianza inunda ahora también las redes sociales. Puesto que los usuarios objeto de estudio claramente muestran una preferencia por ellas para estar informados, analizamos los resultados de la variable 3: Redes sociales más usadas en la Tabla 3, donde encontramos medias, desviaciones típicas y frecuencias en porcentajes. 
RLCS, Revista Latina de Comunicación Social, 79, 67-85

[Investigación] DOI: 10.4185/RLCS-2021-1519| ISSN 1138-5820| Año 2021

Tabla 3. Estadísticos descriptivos para el análisis de las variables $V_{3:}$ Redes sociales más usadas

\begin{tabular}{|l|cc|c|c|c|c|c|c|}
\hline Redes sociales & Media & DT & $\begin{array}{c}\text { Nunca } \\
(\boldsymbol{\%})\end{array}$ & $\begin{array}{c}\text { Poco } \\
(\boldsymbol{\%})\end{array}$ & $\begin{array}{c}\text { Ocasional } \\
(\boldsymbol{\%})\end{array}$ & $\begin{array}{c}\text { Con } \\
\text { frecuencia } \\
(\boldsymbol{\%})\end{array}$ & $\begin{array}{c}\text { Siempre } \\
(\boldsymbol{\%})\end{array}$ & $\begin{array}{c}\mathbf{N} \\
\text { Tik Tok }\end{array}$ \\
\hline Twitter & 2,16 & 1,385 & 48,6 & 18,3 & 11,2 & 12,7 & 9,2 & 465 \\
\hline WhatsApp & 4,85 & 1,479 & 24,7 & 23,9 & 12,9 & 18,7 & 19,8 & 465 \\
\hline Twitch & 1,45 & 0,937 & 0,4 & 6 & 11,8 & 22,2 & 59,6 & 465 \\
\hline YouTube & 3,26 & 1,091 & 2,6 & 26,2 & 20,1 & 35,2 & 15,9 & 465 \\
\hline Facebook & 1,63 & 1,021 & 63 & 21,3 & 8,2 & 4,3 & 3,2 & 465 \\
\hline Instagram & 4,07 & 1,091 & 3,4 & 6,9 & 14,8 & 29 & 45,8 & 465 \\
\hline
\end{tabular}

Fuente: Elaboración propia

Los resultados obtenidos muestran un amplio porcentaje de usuarios que nunca usan Twitch, con un $73 \%(\mathrm{M}=1,45 ; \mathrm{DT}=0,899)$; tampoco emplea Facebook un 63\% $(\mathrm{M}=1,63 ; \mathrm{DT}=1,021)$, y casi la mitad de la muestra $(48,6 \%)$ tampoco utiliza Tik Tok $(\mathrm{M}=2,16$; $\mathrm{DT}=1,385)$. Twitter se muestra como red intermedia con datos muy distribuidos: un 24,7\% (M=2,85; $\mathrm{DT}=1,479)$ nunca lo emplea, aunque un $38,5 \%$ sí lo usa "con frecuencia" o "siempre". En el lado opuesto del consumo de redes se encuentra YouTube con un $51,1 \%(\mathrm{M}=3,26 ; \mathrm{DT}=1,091)$ si se suman las respuestas "con frecuencia" y "siempre"; en el caso de Instagram, el porcentaje se eleva al 74,8\% (M=4,07; DT=1,091), y con WhatsApp el porcentaje sube hasta un $81,8 \%(\mathrm{M}=4,34$; $\mathrm{DT}=0,937)$.

Para el estudio de las variables $4\left(\mathrm{~V}_{4}\right)$ "Tiempo que dedicas a consumir medios" y $5\left(\mathrm{~V}_{5}\right)$ "Percepción de estar informado" se optó por estudiar la correlación de ambas variables con la prueba no paramétrica Rho de Spearman (Rho), por ser la indicada en dos variables categóricas ordinales. Los resultados muestran un valor $\mathrm{Rho}=0.79$, lo que indica que existe una correlación positiva con una fortaleza moderada; sin embargo, el grado de significatividad $\mathrm{p}=.089(\mathrm{p}>0.05)$ indica que la relación no es significativa. Veamos estos datos de modo visual en el Gráfico 3.

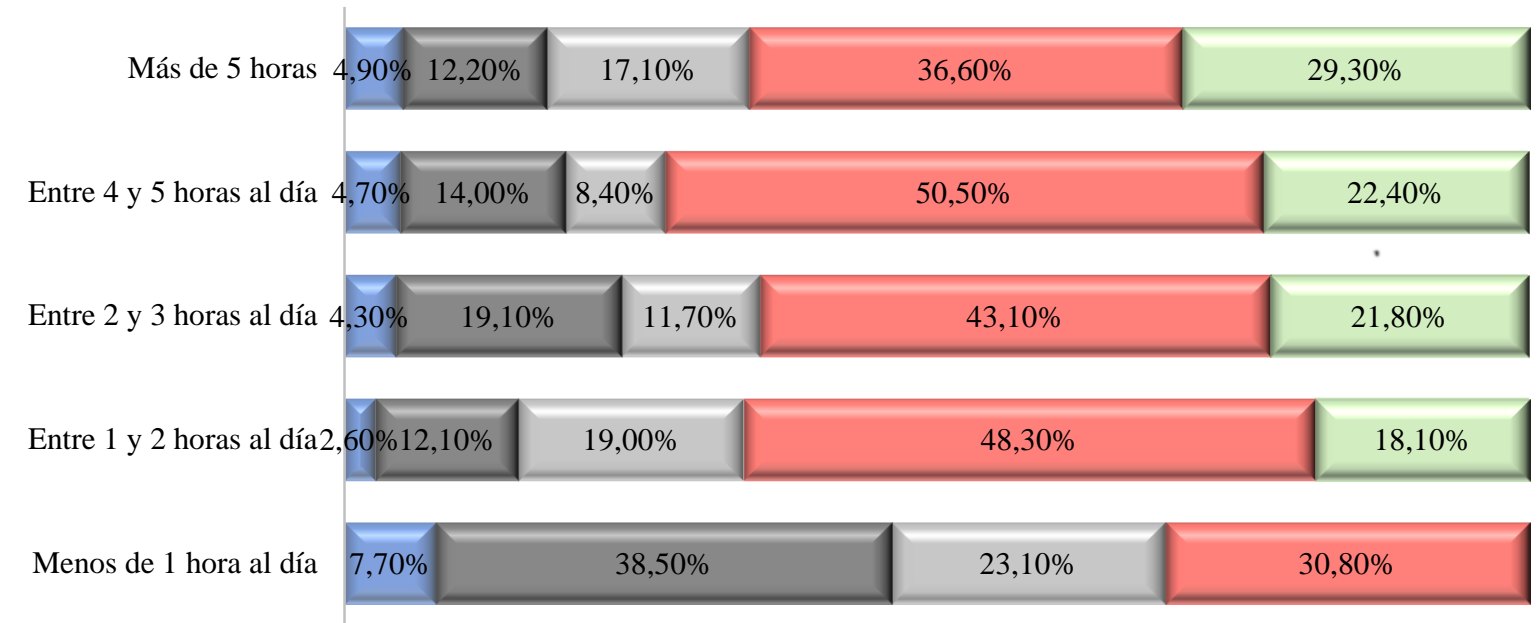

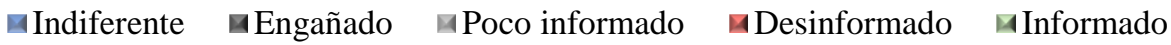

Gráfico 3. Resultados de correlación "Tiempo de consumo" con "Sentimiento con respecto a lo que consumes" 
Fuente: Elaboración propia a partir de la muestra

Aunque estadísticamente no se demuestra una correlación relevante ( $p>0.05)$ entre el tiempo de uso y el sentimiento del usuario, la representación de los datos, a partir de una tabla de contingencia, sí aporta resultados reveladores: el sentimiento de estar informado no obtiene porcentajes elevados incluso en los usuarios que más tiempo pasan conectados, con un 29,39\% como valor máximo en esta categoría. Los resultados más destacables son los porcentajes obtenidos en el número de sujetos que se sienten desinformados independientemente del tiempo que estén conectados. Cabe decir a este respecto que el $97,8 \%$ de la muestra pasa conectado más de una hora al día, el $72,2 \%$ más de dos horas, y un tercio de la muestra más de cuatro horas al día.

\subsection{Consumo, recepción y percepción de fake news}

Los resultados sobre el consumo ofrecen una respuesta a las dos primeras preguntas de investigación; veamos los resultados del segundo constructo para responder a las siguientes (P.I.3- P.I.7). La primera variable se refiere al tipo de información más consumida a partir de la crisis por la COVID19. Como se observa en el gráfico 4, los contenidos más consultados se corresponden con el humor: el $61,8 \%$ los consume mucho y bastante; las enfermedades y la salud resultan también de interés para un $49,2 \%$. El tema más consultado ha sido la política, con un $73,9 \%$ de sujetos que han respondido haberla consumido bastante y mucho. En una línea de interés intermedio se encuentran el entretenimiento y la cultura, con un 39\%, o la alimentación y los cuidados, con un 31,9\% con interés alto por esas temáticas.

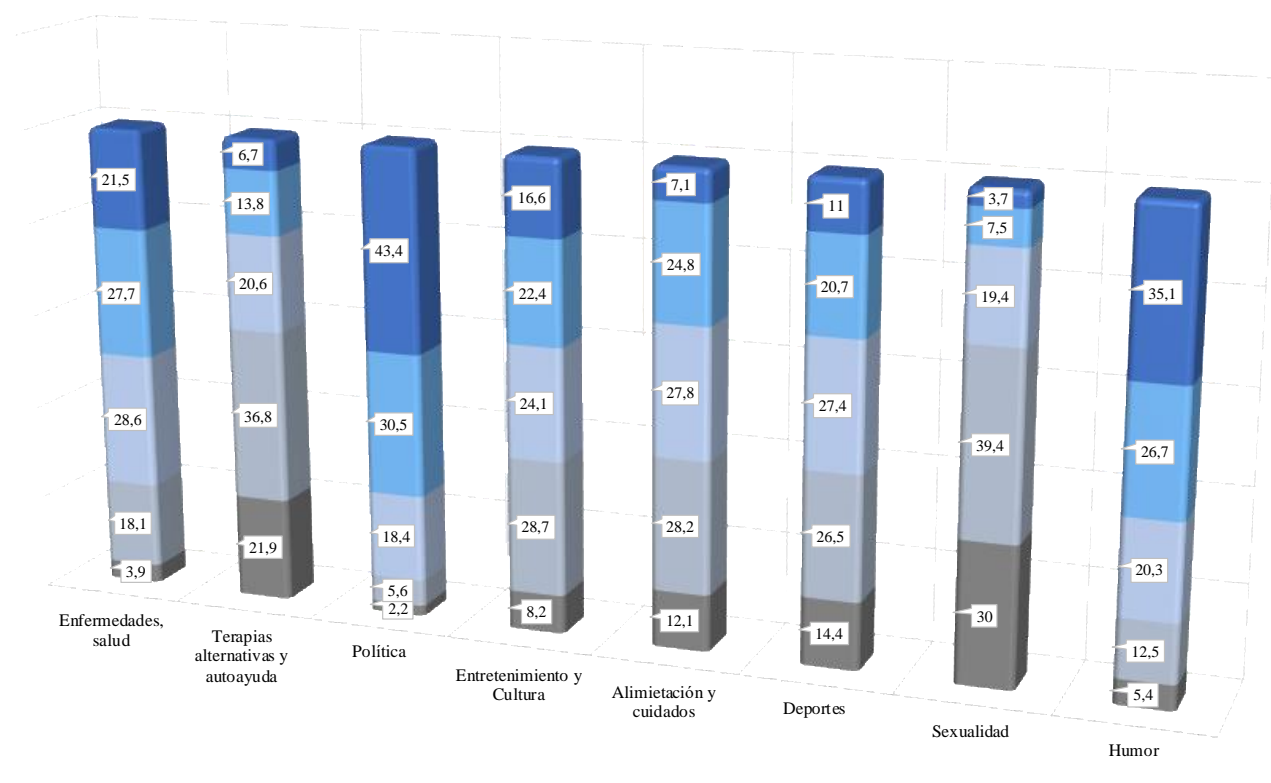

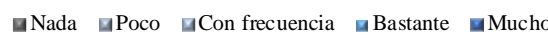

\section{Gráfico 4. Frecuencias de consumo de información por temática en porcentajes}

Fuente: Elaboración propia a partir de la muestra

Otro de los aspectos importantes relacionados con la información que consumen es si son capaces de distinguir las fake news cuando las reciben $\left(\mathrm{V}_{2}\right)$; en este sentido, el $41,7 \%$ declara que sólo las distingue a veces; el 45,8\% las distingue con frecuencia y solo el 11,4\% declara que siempre las distingue. Dado que prácticamente el $100 \%$ de la muestra distingue las noticias falsas, la $\mathrm{V}_{3}$ analiza con qué frecuencia las reciben. El 62,2\% declara que sólo recibe a veces este tipo de contenido, el 
$35,7 \%$ afirma que las recibe con frecuencia o siempre, de modo que también queda explícito que prácticamente la totalidad de la muestra recibe fake news.

En relación con la distinción y recepción de las noticias falsas está el conocimiento de los fact checkers como herramientas válidas para la valoración o reconocimiento de fake news. En este sentido, el 61,1\% de los encuestados declararon no saber qué son, y sólo el 38,7\% afirman tener conocimiento de estos mecanismos. De ellos, sólo el $10 \%$ afirma haber utilizado alguna vez Maldita.es, un 6,7\% ha usado Newtral, un 1,5\% Efe Verifica y un 1,2\% ha usado alguna vez AFP Factual y Chequeado. Estos datos coinciden con estudios recientes sobre el uso de la verificación de noticias en España como los de López Pan y Rodríguez-Rodríguez (2020), en los que se demuestra que el uso de estas herramientas se encuentra todavía en una fase muy incipiente. La Tabla 4 muestra los resultados de esta variable.

Tabla 4. Estadísticos descriptivos para el análisis de las variables $V_{4}$, medios que crees que difunden más fake news

\begin{tabular}{|l|cc|c|c|c|c|c|c|}
\hline Redes sociales & Media & DT & $\begin{array}{c}\text { Nunca } \\
(\boldsymbol{\%})\end{array}$ & $\begin{array}{c}\text { Poco } \\
(\boldsymbol{\%})\end{array}$ & $\begin{array}{c}\text { Con } \\
\text { frecuencia } \\
(\boldsymbol{\%})\end{array}$ & $\begin{array}{c}\text { Bastante } \\
(\boldsymbol{\%})\end{array}$ & $\begin{array}{c}\text { Siempre } \\
(\boldsymbol{\%})\end{array}$ & $\mathbf{N}$ \\
\hline Medios tradicionales & 2,76 & 1,049 & 8,2 & 39,2 & 27,6 & 18,8 & 6,3 & 465 \\
\hline Páginas web & 3,68 & 0,819 & 0,9 & 6,9 & 28,4 & 50,6 & 13,1 & 465 \\
\hline Twitter & 3,74 & 0,855 & 0,2 & 9,1 & 24,1 & 49,4 & 17,2 & 465 \\
\hline Instagram & 3,64 & 0,866 & 0 & 11 & 28,9 & 45,3 & 14,9 & 465 \\
\hline Facebook & 3,88 & 0,853 & 0,2 & 5,8 & 24,6 & 45 & 24,4 & 465 \\
\hline Tik Tok & 3,31 & 1,168 & 8,4 & 17,2 & 23,9 & 35,6 & 14,9 & 465 \\
\hline WhatsApp & 3,96 & 0,931 & 1,1 & 7,1 & 17,7 & 43,3 & 30,8 & 465 \\
\hline Boca a boca & 4,06 & 0,902 & 0,4 & 6 & 17 & 40,1 & 36,4 & 465 \\
\hline
\end{tabular}

Fuente: Elaboración propia

Los resultados obtenidos indican una clara relación con la segunda variable $\left(\mathrm{V}_{2}\right)$ del primer constructo relacionada con la fiabilidad otorgada a los diferentes medios. Los datos sobre percepción asociados a las fake news coinciden con los medios de los que menos se fía esta generación. Si se fija la atención en los datos de las respuestas "Bastante" y "Siempre", observamos que una cuarta parte de la muestra, es decir, 25\% ( $\mathrm{M}=2,76$; $\mathrm{DT}=1,049)$, percibe que los medios tradicionales difunden bastante o siempre noticias falsas. Los datos para el resto de ítems son: páginas web, un 63,7\% (M= 3,68; DT=0,819), Twitter, un 66,6\% $(\mathrm{M}=3,74$; $\mathrm{DT}=0,855)$, Instagram un 60,2\% $(\mathrm{M}=3,64$; $\mathrm{DT}=$ 0,866), Facebook, un 69,4\% (M=3,88; DT=0,853), Tik Tok, un 50,5\% (M=3,31; DT=1,168) y WhatsApp (que era la red más usada por esta generación), un 74,1\% (M=3,96; DT=0,931). Cabe mencionar que el medio que consideran más proclive a la difusión de noticias falsas es el boca a boca con un 76,5\% $(\mathrm{M}=4,06$; $\mathrm{DT}=0,902)$ en los máximos valores.

De los resultados anteriores queda clara la percepción de la Generación $\mathrm{Z}$ de que las redes sociales y el contenido web son los espacios donde más fake news se distribuyen, siendo curiosamente estos los medios que declaran consumir de manera masiva y en los que menos fiabilidad depositan. Analicemos en la siguiente variable $\left(\mathrm{V}_{5}\right)$ qué contenidos creen que contienen más noticias falsas, lo cual se refleja de manera visual en el Gráfico 5. 
RLCS, Revista Latina de Comunicación Social, 79, 67-85

[Investigación] DOI: 10.4185/RLCS-2021-1519| ISSN 1138-5820| Año 2021

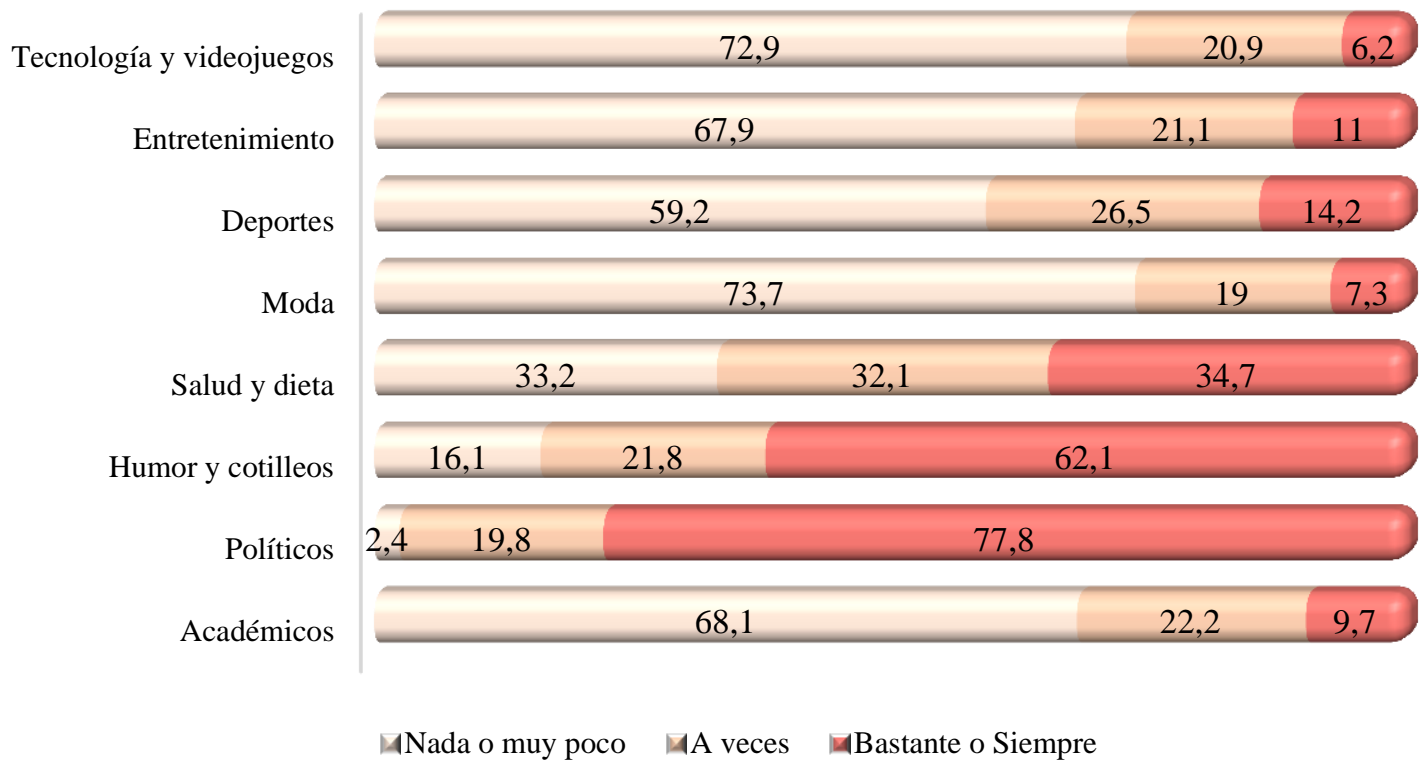

Gráfico 5. Contenidos considerados más propensos a contener fake news

Fuente: Elaboración propia a partir de la muestra

Los resultados muestran un porcentaje elevadísimo en los contenidos políticos, con un 77,8\% de la muestra que considera que son muy propensos a contener noticias falsas, seguidos del humor y el entretenimiento con un $62,1 \%$, y los contenidos de salud con un $34,7 \%$. El resto de temáticas obtiene porcentajes más bajos, pero aún así llama la atención que todos los contenidos generan percepción de noticias falsas, aunque sea en menor medida.

Para finalizar la investigación se midieron las percepciones relacionadas con las fuentes que generan mayor o menor confianza en la muestra objeto de estudio desde que estalló la crisis por la COVID19. Especialmente significativos resultan los datos de desconfianza (Gráfico 6, pirámide roja): el $77,8 \%(\mathrm{M}=1,87 ; \mathrm{DT}=0,985)$ declara no tener ninguna o poca confianza en los políticos; más de la mitad de la muestra 57,9\% (M=2,4; DT=0,802) tampoco muestra confianza en las redes (dato reiterado), y casi la mitad, 46,2\% (M=2,65; DT=0,93) tampoco en los medios de comunicación. La falta de confianza se extiende a un 40,5\% $(\mathrm{M}=2,78$; $\mathrm{DT}=0,919)$ en el caso de los periodistas o comunicadores.

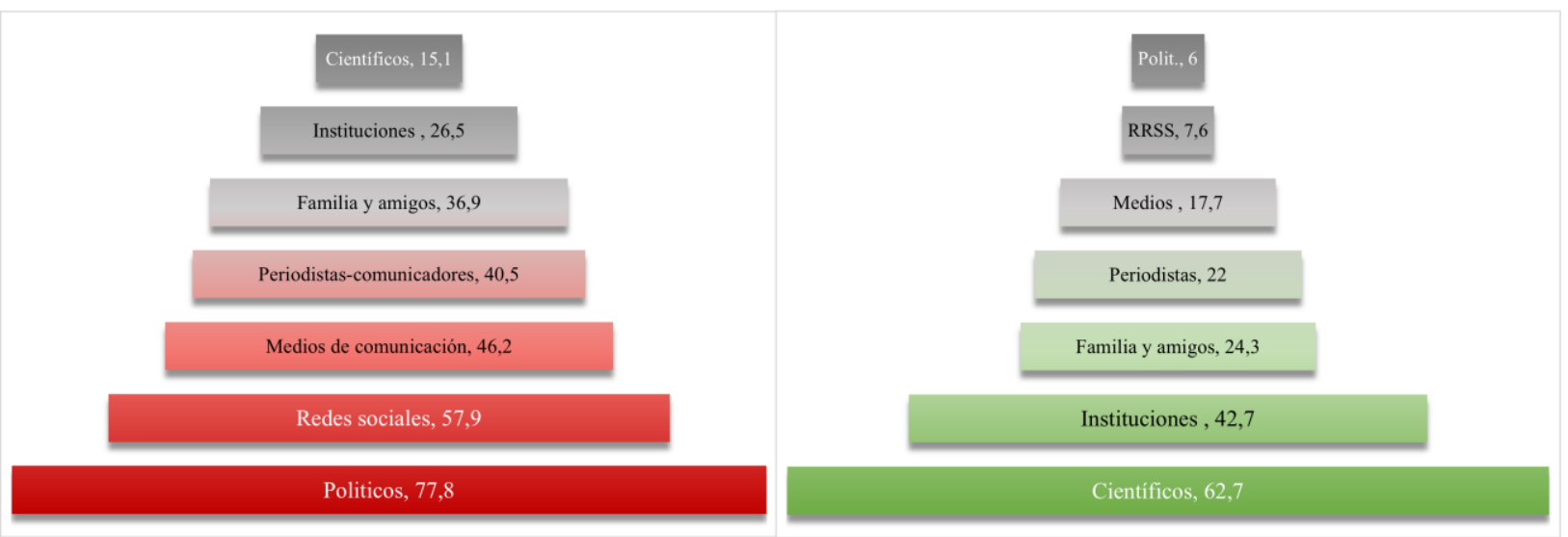

Gráfico 6. Representación de los medios que menos y más confianza ofrecen a los jóvenes desde el estallido de la crisis por Covid-19 (en porcentajes) 
Fuente: Elaboración propia a partir de la muestra

Los datos de la derecha (Gráfico 6, pirámide verde) muestran los resultados de la confianza, el 62,7\% $(\mathrm{M}=3,63 ; \mathrm{DT}=1,018)$ de los sujetos confían en los científicos, y apenas la mitad de la muestra, el 42,7\% (M= 3,24; DT= 1,102) lo hace en las instituciones. La confianza en los medios y los periodistas queda en un $17,7 \%$ y un $22 \%$ respectivamente, lo que indica que la confianza de la generación $\mathrm{Z}$ se encuentra ciertamente en niveles bajos.

\section{Discusión y conclusiones}

Los resultados de la investigación permiten concluir que existe una preocupante falta de confianza de los jóvenes hacia los principales actores comunicativos: medios, periodistas o comunicadores y redes sociales. Los medios y los periodistas han perdido su legitimidad como gatekeepers de la información frente a una generación $\mathrm{Z}$ que consume contenido masiva e intensamente a través de las redes sociales (Jarvis, 2015; Pérez-Tornero, 2020). Aunque una de las limitaciones importantes del estudio es la falta de representatividad en la muestra, se recogen evidencias relevantes tanto para las universidades que forman en Comunicación como para los medios y periodistas, pues ésta es y será su audiencia. En línea con los trabajos de Álvarez Ramos et al. (2019), Gutiérrez-García y BarriosRubio (2021), Boulianne y Theocharis (2020), Banks et al. (2020), Gómez Calderón et al. (2020) o Pérez-Escoda et al. (2021), reconocer los hábitos y comportamientos de la audiencia joven, así como sus preferencias, constituye el único modo que tiene el periodismo de responder a sus demandas y volver a legitimarse como actor social.

Respecto al primer constructo de estudio, cabe apuntar que, para sentirse informada, esta generación recurre a sus medios nativos, los digitales, salvo en el caso de la televisión, que curiosamente mantiene un grado aceptable de consumo en la mitad de la muestra. La fiabilidad que otorgan a los medios es inversamente proporcional al consumo que hacen de ellos: afirman que apenas consumen radio ni en un receptor analógico ni en dispositivos online, tampoco prensa tradicional, y sin embargo son los medios en los que más confianza depositan. No confían en las redes o en los contenidos en streaming, pero sí los consumen de modo masivo; sólo la televisión y la prensa online mantienen niveles similares de consumo y fiabilidad.

La confiabilidad de la ciudadanía ha sido estudiada en numerosos trabajos cuyos resultados mostraban una polarización de opiniones y sentimientos (Aruguete et al., 2020; Banks et al., 2020; Masip et al., 2020); sin embargo, las evidencias de este estudio ponen de manifiesto un problema distinto: la falta de confianza, el descrédito que otorga esta generación a los medios que consume, lo que subraya la disonancia existente entre lo que los periodistas comunican y las expectativas de la audiencia en línea ya constatada en los estudios de Loosen et al. (2020). En contra de otros informes recientes como el de Negredo et al. (2020) en el Digital News Report.es 2020, Facebook se muestra como una de las redes menos usadas por esta generación, que parece hallarse masivamente en Instagram y en Whatsapp. Su exposición en tiempo es alta -prácticamente tres cuartas partes de la muestra están conectados al día más de dos horas-, y el sentimiento que más les inunda es el de estar desinformados, seguido del de estar poco informados o incluso engañados.

El segundo constructo genera nuevas conclusiones enlazadas con las anteriores. Desde la explosión de la crisis por la COVID-19, el tema que más interesa es la política: prácticamente tres cuartas partes de la muestra muestran un interés alto o muy alto, seguida por el humor. Respecto a las noticias falsas, declaran distinguirlas sólo la mitad de los encuestados, aunque el $97 \%$ de ellos afirma que las recibe. Una de las alternativas más eficaces a esta problemática, los fact checkers, son grandes desconocidos para esta generación: sólo el 40\% declaró conocer qué eran, y de ellos sólo el 
19\% había usado aluna vez uno. En este sentido, entre las principales conclusiones de este estudio destaca que el fact checking como variante de verificación del periodismo en la era digital todavía no puede considerarse como una solución a la problemática de la desinformación debido a su baja penetración de uso y conocimiento entre los usuarios, como demuestran otros estudios (López Pan y Rodríguez-Rodríguez, 2020).

Otra llamativa paradoja es que, siendo la política y el humor los contenidos que más les interesan, los consideran al mismo tiempo los más propensos a contener fake news. La conclusión más alarmante es la desconfianza abrumadora hacia los medios y los periodistas, que adquiere un tono alarmante en el caso de los políticos y las redes sociales. Se subraya así la alerta subyacente de un sistema en quiebra y una crisis profunda para los países democráticos basados en la libertad de expresión y pensamiento: una población que desconfía de los medios y de los políticos es una población polarizada, fácilmente manipulable e incapaz de tomar decisiones libres e informadas (Aruguete at al., 2020).

Teniendo en cuenta la base sobre la que se articula este estudio, en la que la aceptación de un modelo de consumo afecta la construcción de la propia identidad a partir de la exposición a informaciones y estímulos audiovisuales en un tiempo, un lugar y a través de unos dispositivos o medios físicos, los resultados confieren una relevancia significativa a los retos del periodismo actual. Esto implica asumir puntos de vista, razonamientos, estéticas y valores para una construcción cultural (Couldry et al., 2007; Pérez-Tornero, 2020). Podemos alfabetizar a los jóvenes (García-Ruiz y Pérez-Escoda, 2021) y darles herramientas de fact cheking, pero si luego no confían, si no dan crédito a las fuentes, si no encuentran legitimidad en los discursos mediáticos, esas medidas no serán efectivas. Si unimos estas reflexiones a las conclusiones ya argumentadas, resulta irrebatible que el periodismo necesita conocer mejor a su audiencia, entenderla, identificar sus demandas y aprovechar las ventajas de la red para contrarrestar sus peligros. La adecuación entre la información que se comunica y la realidad, así como la conexión con las expectativas de quienes la consumen, se impone como una necesidad sustantiva e inaplazable para el periodismo en el reto de legitimar su discurso en las redes sociales.

\section{Referencias}

Álvarez Ramos, E., Heredia Ponce, H. y Romero Olive, M. (2019). La Generación Z y las Redes Sociales. Una visión desde los adolescentes en España. Revista Espacios, 40(20), 1-13. https://www.revistaespacios.com/a19v40n20/a19v40n20p09.pdf

Aruguete, N. Calvo, E. y Ventura, T. (2020), News Sharing, Gatekeeping, and Polarization: A Study of the \#Bolsonaro Election. Digital Journalism, 9(1), 1-23. https://doi.org/10.1080/21670811.2020.1852094

Banks, A., Calvo, E., Karol, D. y Telhami, S. (2020). \#PolarizedFeeds: Three Experiments on Polarization, Framing, and Social Media. The International Journal of Press/Politics. https://doi.org/10.1177/1940161220940964

Barrios-Rubio, A. y Pedrero-Esteban, L.M. (2021). The transformation of the Colombian Media industry in the Smartphone Era. Journal of Creative Communications, 16(1), 45-60. https://doi.org/10.1177/0973258620981347

Boulianne, S. y Theocharis, Y. (2020). Young people, digital media, and engagement: A metaanalysis of research. Social Science Computer Review, 38(2), pp. 111-127. https://doi.org/10.1177/0894439318814190 
Casero-Ripollés, A. y García-Gordillo, M. (2020). La influencia del Periodismo en el ecosistema digital. L.M. Pedrero-Esteban y A. Pérez-Escoda, Cartografía de la comunicación postdigital: Medios y audiencias en la Sociedad de la COVID-19. Navarra, España: Aranzadi-Thomson Reuters, pp. 157-176.

Castillo-Abdul, B., Romero-Rodríguez, L.M. y Larrea-Ayala, A. (2020). Kid influencers in Spain: understanding the themes they address and preteens' engagement with their YouTube channels. Heliyon, 6(9), 1-10. https://doi.org/10.1016/j.heliyon.2020.e05056

Comisión Europea (2018). Comunicación de la Comisión al Parlamento Europeo, al Consejo, al Comité Económico y Social Europeo y al Comité de las Regiones, sobre La lucha de la desinformación en línea: un enfoque europeo. COM/2018/236 final.

Couldry, N., Livingstone, S. y Markham, T. (2007). Media consumption and Public engagement. Palgrave McMillan.

Dessart, L. (2017). Social media engagement: a model of antecedents and relational outcomes. Journal of Marketing Management, 33, 375-399. https://doi.org/10.1080/0267257X.2017.1302975

Elías, C. (2018). Fakenews, poder y periodismo en la era de la posverdad y 'hechos alternativos'. Revista Ámbitos, 40. https://institucionales.us.es/ambitos/fakenews-poder-y-periodismo-en-la-erade-la-posverdad-y-hechos-alternativos/

Espiritusanto, O. y Gonzalo Rodríguez, P. (2011). Periodismo ciudadano. Evolución positiva de la comunicación. Ariel y Fundación Telefónica.

European Commission (2019). Media Use in the European Union. Report. Fieldwork Autumn 2019. https://op.europa.eu/en/publication-detail/-/publication/c2fb9fad-db78-11ea-adf701aa75ed71a1/language-en

European Commission (2020). Standard Eurobarometer 93. Summer 2020. Report. Public Opinion in the European Union. https://ec.europa.eu/commfrontoffice/publicopinion/index.cfm/ResultDoc/download/DocumentKy $\underline{191061}$

Farkas, J. y Schou, J. (2020). Post-truth, fake news and democracy. New York, Routledge.

Galloway, S. (2018). Four. El ADN secreto de Amazon, Apple, Facebook y Google. Madrid: Conecta.

García-Ruiz, R. y Pérez-Escoda, A. (2021). La competencia digital docente como clave para fortalecer el uso responsable de Internet. Campus Virtuales, 10(1), 59-71. http://www.uajournals.com/ojs/index.php/campusvirtuales/article/view/781Com

Gómez Calderón, B., Córdoba-Cabús, A. y Méndez Nieto, A. (2020). Jóvenes y fake news. Un análisis sociodemográfico aplicado al caso andaluz. Revista Científica de Información y Comunicación, 17, 481-501. Disponible en: https://tinyurl.com/3nj4tu38 
Gutiérrez-García, M. y Barrios-Rubio, A. (2021). Prácticas juveniles de consumo sonoro, entre grandes plataformas y el ecosistema radiofónico: caso Colombia-España. Comunicación y Sociedad, 18, 1-24. https://doi.org/10.32870/cys.v2021.7820

Jarvis, J. (2015). El fin de los medios de comunicación de masas: ¿Cómo serán las noticias del futuro? Barcelona: Grupo Planeta.

Jenkins, H. (2008). Convergence Culture. La cultura de la convergencia de los medios de comunicación. Barcelona: Paidós Comunicación.

Keyes, R. (2004). The post-truth era: Dishonesty and deception in contemporary life. New York: St. Martin's Press. ISBN: 9780312306489

Larrondo-Ureta, A., Peña-Fernández, S. y Agirreazkuenaga-Onaindia, I. (2020). Hacia una mayor participación de la audiencia: experiencias transmedia para jóvenes. Estudios sobre el mensaje periodístico, 26(4), 1445-1454. https://doi.org/10.5209/esmp.71375

Loosen, W., Reimer, J. y Hölig, S. (2020). What journalists want and what they ought to do (in)congruences between journalists' role conceptions and audiences' expectations. Journalism studies, 21(12), 1744-1774. https://doi.org/10.1080/1461670X.2020.1790026

López Pan, F. y Rodríguez Rodríguez, J. (2020). El Fact Checking en España. Plataformas, prácticas y rasgos distintivos. Estudios Sobre El Mensaje Periodístico, 26(3), 1045-1065. https://doi.org/10.5209/esmp.65246

Masip, P., Suau J. y Ruiz-Caballero, C. (2020). Perceptions on media and disinformation: Ideology and polarization in the Spanish media system. Profesional de la Información, 29(5), 1-13. https://doi.org/10.3145/epi.2020.sep.27

Masip, P., Suau, J. y Ruiz-Caballero, C. (2017). Questioning the selective exposure to news: Understanding the impact of social networks on political news consumption. American behavioral scientist, 62(3), 300-319. https://doi.org/10.1177/0002764217708586

Mendiguren, T., Pérez Dasilva, J. y Meso Ayerdi, K. (2020). Actitud ante las Fake News: Estudio del caso de los estudiantes de la Universidad del País Vasco. Revista de Comunicación, 19(1), 171 184. https://doi.org/10.26441/RC19.1-2020-A10

Negredo, S., Amoedo, A., Vara Miguel, A., Moreno, E. y Kaufmann, J. (2020). Digital News Report.es 2020. https://www.digitalnewsreport.es/2020/gobiernos-politicos-y-redes-socialesprincipales-responsables-del-auge-de-los-bulos/

Newman, N. y Fletcher, R. (2017). Bias, Bullshit and Lies: Audience Perspectives on Low Trust in the Media. Reuters Institute and University of Oxford. https://doi.org/10.2139/ssrn.3173579

Newman, Ni., Fletcher, R., Schulz, A. Andi, S., Nielsen, R. (2020). Digital news report 2020. Reuters Institute. https://reutersinstitute.politics.ox.ac.uk/sites/default/files/202006/DNR_2020_FINAL.pdf

Nielsen, R. K. y Graves, L. (2017). News You Don't Believe: Audience Perspectives on Fake News. Oxford: Reuters Institute for the Study of Journalism. 
https://reutersinstitute.politics.ox.ac.uk/sites/default/files/2017-

10/Nielsen\%26Graves_factsheet_1710v3_FINAL_download.pdf

Pariser, E. (2011). The filter bubble. How the new personalized web is changing what we read and how we think. London: Penguin Books.

Paulussen S., Domingo D., Heinonen A., Singer J., Quandt T. y Vujnovic M. (2008). Citizen participation in online news media. An overview of current developments in four European countries and the United States. In: Quandt T., Schweiger W. (eds) Journalismus online Partizipation oder Profession? VS Verlag für Sozialwissenschaften. https://doi.org/10.1007/978-3$\underline{\text { 531-91094-9_18 }}$

Pérez-Curiel, C. y Velasco Molpeceres, A. M. (2020). Impacto del discurso político en la difusión de bulos sobre Covid-19. Influencia de la desinformación en públicos y medios. Revista Latina, 78, 65-97. https://doi.org/10.4185/rlcs-2020-1469

Pérez-Escoda, A., Pedrero-Esteban, L.M., Rubio-Romero, J. y Jiménez-Narros, C. (2021). Fake News Reaching Young People on Social Networks: Distrust Challenging Media Literacy. Publications 2021, 9(2) 24. https://doi.org/10.3390/publications9020024

Pérez-Escoda, A., Jiménez-Narros, C., Perlado-Lamo-De-Espinosa, M. y Pedrero-Esteban, L.M. (2020). Social Networks' Engagement During the COVID-19 Pandemic in Spain: Health Media vs. Healthcare Professionals. International Journal of Environmental Research and Public Health, 17, 5261, https://doi.org/10.3390/ijerph17145261

Pérez-Maíllo, A.; Sánchez-Serrano, C.; y Pedrero-Esteban, L.M. (2018). Viaje al Centro de la Radio. Diseño de una experiencia de alfabetización transmedia para promover la cultura radiofónica entre los jóvenes. Comunicación y Sociedad, no 33, 171-20. https://doi.org/10.32870/cys.v0i33.7031

Pérez-Tornero, J.M. (2020). La gran mediatización. El tsunami que expropia nuestras vidas. Barcelona: UOC.

Romero-Rodríguez, L. M., Tejedor, S. y Castillo-Abdul, B. (2021). From the Immediacy of the Cybermedia to the Need for Slow Journalism: Experiences from Ibero-America. Journalism Practice, 1-19. https://doi.org/10.1080/17512786.2020.1870530

Sádaba-Chalezquer, C. y Pérez-Escoda, A. (2020). La generación 'streaming' y el nuevo paradigma de la comunicación digital. L.M. Pedrero-Esteban y A. Pérez-Escoda, Cartografía de la comunicación postdigital: Medios y audiencias en la Sociedad de la COVID-19. Navarra: Aranzadi-Thomson Reuters, pp. 97-114.

Tavakol, M., and Dennick, R. (2011). Making sense of cronbach's alpha. International Journal of Medical Education, 2, 53-55. https://doi.org/10.5116/ijme.4dfb.8dfd

Van Dijk, J. (2013). The culture of Connectivity. A critical History of Social Media. Oxford: Oxford University Press.

Vilches, L. (2011). La Investigación en Comunicación. Métodos y Técnicas en la era Digital. Barcelona: GEDISA. 
RLCS, Revista Latina de Comunicación Social, 79, 67-85

[Investigación] DOI: 10.4185/RLCS-2021-1519| ISSN 1138-5820| Año 2021

We Are Social (2021). Digital Report 2021. El informe de las tendencias digitales. https://wearesocial.com/es/blog/2021/01/digital-report-2021-el-informe-sobre-las-tendenciasdigitales-redes-sociales-y-mobile

Webster, J. G. (2014). The Marketplace of Attention: How Audiences Take Shape in the Digital Age. Cambridge, MA: The MIT Press.

WHO (2020). Managing the COVID-19 Infodemic: Promoting Healthy Behaviours and Mitigating the Harm from Misinformation and Disinformation. https://www.who.int/news/item/23-09-2020

\section{AUTORES:}

\section{Ana Pérez-Escoda}

Profesora e investigadora en el grupo Innovación en Comunicación y Medios (Nebrija Innomedia) en la Facultad de Comunicación y Artes de la Universidad Antonio de Nebrija. Miembro de diferentes proyectos de I+D nacionales y europeos, sus líneas de investigación se centran en alfabetización digital, competencias digitales y comunicación digital, con especial atención a las redes sociales. Autora del libro Alfabetización mediática, TIC y competencias digitales (UOC) y coeditora de Cartografía de la Comunicación postdigital. Medios y audiencias en la sociedad de la COVID-19 (Aranzadi), así como de numerosos artículos de investigación en revistas nacionales e internacionales. Ha sido coordinadora de Posgrado y coordinadora de innovación docente en la UPSA (2017-2020) y editora asociada de la revista Comunicar JCR Q1 (2017-2020).

Índice H: 15

Orcid ID: http://orcid.org/0000-0002-4895-0043

Google Scholar: https://scholar.google.com/citations?user=vo89GUMAAAAJ\&hl=es

ResearchGate: https://www.researchgate.net/profile/Ana_Perez_escoda

Scopus ID: https://www.scopus.com/authid/detail.uri?authorId=57191505785

Academia.edu: https://www.nebrija.com/medios/innomedia/equipo/

\section{Luis Miguel Pedrero Esteban}

Catedrático e investigador principal del grupo Innovación en Comunicación y Medios en la Facultad de Comunicación y Artes de la Universidad Antonio de Nebrija. Ha sido redactor de informativos en la Cadena SER, guionista de programas musicales en Los 40; colaborador en COPE y Onda Cero; y consultor de Radio Blanca. Autor de los libros La radio musical en España: historia y análisis; La industria audiovisual y publicitaria en Castilla y León (con Ángel Badillo y Marta Fuertes) y La televisión y los niños (con Javier Muñoz). Coeditor de La transformación digital de la radio (con José $\mathrm{M}^{\mathrm{a}}$ García-Lastra) y de Cartografía de la Comunicación postdigital. Medios y audiencias en la sociedad de la COVID-19 (con Ana Pérez Escoda). Editor del semanal digital 'Pantallas, Ondas, Frames y Bits'. Miembro de la Junta Directiva de la Sociedad Española de Periodística.

Índice H: 14

Orcid ID: https://orcid.org/0000-0003-4949-2360

Google Scholar: https://scholar.google.com/citations?user=Q8UU05MAAAAJ\&hl=es

ResearchGate: https://www.researchgate.net/profile/Luis_Pedrero_Esteban

Scopus ID: $h$ ttps://www.scopus.com/authid/detail.uri?authorId=56925350600

Academia.edu: https://nebrija.academia.edu/LuisMiguelPedreroEsteban 